06

\title{
Эффекты насыщения и самонасыщения в спектроскопии однонаправленных волн на переходы между уровнями с полным моментом $J=1$
}

\author{
(C) Э.Г. Сапрыкин ${ }^{1}$, А.А. Черненко ${ }^{2}$ \\ ${ }^{1}$ Институт автоматики и электрометрии Сибирского отделения РАН, \\ 630090 Новосибирск, Россия \\ ${ }^{2}$ Институт физики полупроводников Сибирского отделения РАН, \\ 630090 Новосибирск, Россия \\ e-mail: chernen@isp.nsc.ru
}

Поступила в редакцию 13.07.2017 г.

В окончательной редакции 04.06.2019 г.

Принята к публикации 11.06.2019 г.

\begin{abstract}
Аналитически и численно исследовано нелинейное поглощение пробного поля в системе вырожденных уровней атома с полным моментом $J=1$ в однонаправленных волнах при учете насыщения собственным спонтанным излучением. Установлены закономерности из менения амплитудных и частотных характеристик нелинейного резонанса на открытом и закрытом типах переходов при разных поляризациях волн, вносимые самонасыщенияем. Выявлены специфические проявления эффекта, включая и связанные с магнитной когерентностью уровней.
\end{abstract}

Ключевые слова: самонасыщение, нелинейный резонанс, поляризация.

DOI: $10.21883 /$ OS.2019.10.48375.164-17

\section{1. Введение}

В работе [1] было продолжено развитие теории нелинейных оптических явлений в атомных системах при одновременном воздействии лазерного когерентного излучения и собственного спонтанного излучения (СИ) протяженной системы. В рамках развитой модели самонасыщения под действием СИ [2] исследованы спектроскопические проявления данного эффекта в спектроскопии однонаправленных волн на примере простых двух- и трехуровневых невырожденных атомных переходов. Показано, что в случае однонаправленных волн в эффекте СИ на таких переходах проявляются закономерности, установленные в методе пробного поля при встречно направленных волнах $[3,4]$. Такие как изменение параметров селективных структур Беннета (их сужение) в распределении атомов по скоростям и формирование однородной полосы насыщения поля пробной волны, приводящего к изменению амплитуды крыла линии и параметров населенностной части нелинейного резонанса. При этом в случае однонаправленных волн значительный вклад в форму резонансов насыщенного поглощения вносят нелинейные интерференционные эффекты (НИЭФ), приводящие к образованию в спектрах резонансов узких структур - пика просветления либо пика поглощения [5-9]. Ширины этих структур могут быть сравнимыми с частотами вынужденных переходов, индуцируемых самонасыщением. Это означает, что в условиях однонаправленных волн действие самонасыщения может быть более выраженным по сравнению со случаем встречных волн. При этом оказывается возможным непосредственное измерение частот СИ-переноса [1].

Модель атома с невырожденными уровнями может применяться при описании реальных многоуровневых систем, но с ограничениями, зависящими от состояний атома и поляризации излучения. В этой связи представляется важным исследование проявления эффекта СИ в спектрах насыщенного поглощения однонаправленных волн в более сложной модели атома, в частности, в системе двух вырожденных уровней с полным моментом $J=1$. На данном переходе форма нелинейного резонанса качественным образом зависит от взаимной ориентации поляризаций сильной и пробной волн, а в образование узких структур резонанса значительный вклад наряду с НИЭФ может вносить магнитная когерентность уровней, индуцируемая оптическими полями волн линейной поляризации.

Стандартным приемом анализа вырожденной атомной системы, взаимодействующей с резонансным лазерным излучением, является разбиение ее на двухуровневые подсистемы, связанные между собой спонтанными и вынужденными переходами. В результате система кинетических уравнений, описывающих процесс взаимодействия, оказывается даже без учета когерентности магнитных подуровней системой порядка $2 M(M-$ магнитное квантовое число уровня). Получение точных аналитических решений такой системы уравнений сопряжено с серьезными трудностями, поэтому при решении используются приближения по ряду параметров. Однако такой подход не позволяет детально проследить за динамикой отклика системы на вариации параметров, 
используемых в опытах (интенсивностей оптических полей, взаимной ориентации их поляризаций и т.д.). Возможности современных вычислительных методов в большой степени снимают перечисленные трудности и позволяют провести анализ системы кинетических уравнений с высокой степенью точности для произвольных значений интенсивностей как насыщающего, так и пробного полей, ориентации их поляризаций, полных моментов нижнего и верхнего состояний атома. Подобный подход с использованием аналитических и численных решений исходных уравнений реализован нами в настоящей работе применительно к атомному переходу между уровнями с полным моментом $J=1$.

\section{2. Спектр поглощения пробного поля при самонасыщении в системе вырожденных уровней с полным моментом $J=1$}

Будем рассматривать задачу о спектре поглощения пробного поля на атомном переходе между уровнями с полным моментом $J=1$ в присутствии излучения насыщающего поля сильной волны. Сильная волна предполагается плоской, монохроматической, линейно поляризованной (частота $\omega$, волновой вектор $\mathbf{k}$, напряженность электрического поля $\mathbf{E}$ ), резонансной атомному переходу $m-n$ (частота перехода $\omega_{m n}$ ). Пробная волна также монохроматическая (частота $\omega_{\mu}$, волновой вектор $\mathbf{k}_{\mu}$, напряженность электрического поля $\mathbf{E}_{\mu}$ ), поляризована либо параллельно, либо ортогонально поляризации сильного поля и направлена вдоль направления распространения сильной волны. Газ считаем достаточно разреженным, чтобы можно было пренебречь столкновениями. Предполагается, что на среду накладывается постоянное магнитное поле с напряженностью Н, направление которого задает ось квантования в атоме. Будем интересоваться влиянием СИ на форму линии поглощения пробной волны.

При решении задачи будем исходить из кинетических уравнений для матрицы плотности атомной системы в модели релаксационных констант [5]. В этом случае динамика диагональных элементов матрицы плотности $\left(\rho_{i}=\rho_{i i}\right)$ с учетом членов, описывающих СИ в модели [2-4], определяется следующей системой уравнений:

$$
\begin{aligned}
& \frac{d \rho_{i}}{d t}+\Gamma_{i} \rho_{i}=Q_{i}+\sum_{k} A_{k i} \rho_{k}-\sum_{k}\left(\rho_{i}-\rho_{k}\right) \\
& -2 \operatorname{Re}\left(i \sum_{j} V_{i j} \rho_{i j}\right)-2 \operatorname{Re}\left(i \sum_{j} \sum_{j} V_{i j}^{\mu} \rho_{j i}\right) .
\end{aligned}
$$

Для недиагональных элементов матрицы плотности справедлива следующая система уравнений:

$$
\frac{d \rho_{i k}}{d t}+\left(\Gamma_{i k}+i \omega_{i k}\right) \rho_{i k}=-i[V, \rho]_{i k}=-i\left[V^{\mu}, \rho\right]_{i k}+R_{i k}^{(2)} .
$$

В уравнениях (1), (2) оператор полной производной есть $\frac{d}{d t}=\frac{\partial}{\partial t}+\mathbf{v} \nabla, \Gamma_{i}-$ константы радиационной релаксации уровней по всем каналам распада, включая уход на третьи уровни, $\Gamma_{i k}$ - полуширины линий переходов, $Q_{i}$ - скорости возбуждения уровней перехода, обеспечивающие их заселенность в отсутствие сильного и пробного полей (столкновения с электронами плазмы разряда и спонтанный распад вышележащих уровней). Величины $V$ и $V^{\mu}$ - операторы взаимодействия атома с сильным и пробным полем в дипольном приближении, определяемые соответственно как

$$
\begin{gathered}
V=-G \exp (i(\mathbf{k r}-\omega t))+\text { э. c., } \\
V^{\mu}=-G^{\prime \prime} \exp \left(i\left(\mathbf{k}_{\mu} \mathbf{r}-\omega_{\mu} t\right)\right)+\text { э. с., }
\end{gathered}
$$

где операторы $G=\mathbf{d E} / 2 \hbar, G^{\mu}=\mathbf{d E}_{\mu} / 2 \hbar$, a $\mathbf{d}$ - оператор дипольного момента. Слагаемые $A_{k i} \rho_{k}$ в системе уравнений (1) определяют спонтанный распад $k$-го подуровня верхнего $m$-состояния на $i$-й подуровень нижнего $n$ состояния (скорости данного процесса определяются первым коэффициентом Эйнштейна $A_{m n}$ и коэффициентами векторного сложения для переходов между этими подуровнями). Они присутствуют в уравнениях для населенностей нижних уровней и отсутствуют в уравнениях для населенностей верхних уровней. Слагаемые $\sum v_{i k}\left(\rho_{i}-\rho_{k}\right)$ описывают переходы, индуцируемые полем спонтанного излучения возбужденных атомов (эффект СИ). Величина $v_{i k} \equiv v_{n m}^{M^{\prime} M}$ определяет частоту индуцированных переходов между магнитными подуровнями нижнего $n$ - и верхнего $m$-состояний. Согласно модели [2], суммарная по подуровням частота самонасыщения на переходе определяется как

$$
v_{n m}=\sum_{M^{\prime} M} v_{n m}^{M^{\prime} M}=g_{n m}\left\langle\rho_{m}\right\rangle,
$$

где $\left\langle\rho_{m}\right\rangle$ - полная плотность частиц в возбужденном состоянии, а величина $g_{n m}=\left(\lambda_{m n} A_{m n} / 2\right)^{2} d_{\mathrm{ef}} / k v_{T}$, где $\lambda_{m n}$ - длина волны, $A_{m n}-$ первый коэффициент Эйнштейна атомного перехода, $d_{\mathrm{ef}}-$ эффективный поперечный размер области взаимодействия, ограниченный условием оптически тонкой среды, $k-$ волновое число сильного поля, $v_{T}-$ наиболее вероятная скорость частиц ансамбля. Появление слагаемых, обусловленных СИ, в уравнениях для диагональных элементов приводит, согласно [4], к изменению констант релаксаций $\Gamma_{m}, \Gamma_{n}$ уровней: $\Gamma_{n} \rightarrow \Gamma_{n}+v_{n m}, \Gamma_{m} \rightarrow \Gamma_{m}+v_{n m}$. Оценки показывают, что эффект СИ может изменять константы релаксации уровней атомов в газовой среде, особенно метастабильных. Так, при $\lambda_{m n}=6 \cdot 10^{-5} \mathrm{~cm}$, $d_{\mathrm{ef}}=0.5 \mathrm{~cm}, k v_{T}=5 \cdot 10^{9} \mathrm{~s}^{-1}, A_{m n}=3 \cdot 10^{7} \mathrm{~s}^{-1}$ имеем $g_{\text {nm }} \approx 10^{-4} \mathrm{~cm}^{3} \mathrm{~s}^{-1}$. При плотности возбужденных частиц $\left\langle\rho_{m}\right\rangle=10^{10} \mathrm{~cm}^{-3}$ значение частоты $v_{n m} \approx 10^{6} \mathrm{~s}^{-1}$ превосходит скорость релаксации метастабильных уровней. Зависимость частоты самонасыщения $v_{n m}$ от полной населенности верхнего состояния приводит к нелинейному характеру стационарной системы уравнений. При 
решении системы уравнений возникает параметр $a_{m n}$, определяемый как $a_{m n}=g_{n m} N_{n m} / \Gamma_{m}$, где $N_{n m}-$ разность заселенностей $n$ - и $m$-состояний в отсутствие оптических полей. Параметр $a_{m n}$ характеризует относительную частоту самонасыщения перехода, его максимальное значение в модели [2] может достигать величины $\sim 2$.

В уравнения (2) включены слагаемые $R_{i k}^{(2)}$, определяющие спонтанный перенос индуцируемой оптическими полями магнитной когерентности подуровней состояния $m$ (недиагональные элементы матрицы плотности $\rho_{i k}$ между подуровнями этого состояния) на нижнее $n$-состояние. Далее теоретическое рассмотрение проведем в стационарном случае для переходов между возбужденными $m$ - и $n$-состояниями атома, для которых в стационарных решениях уравнений (1),(2) в пространственно ограниченных полях можно пренебречь производными по поперечной координате. Это справедливо для состояний атома, время жизни которых меньше либо сравнимо со средним временем пролета атомом светового пучка, т. е. $1 / \Gamma_{n} \leq d / v_{T}$. При поперечном размере светового пучка $d \sim 0.1-1 \mathrm{~cm}$ и скорости $v_{T}=10^{4} \mathrm{~cm} / \mathrm{s}$ имеем оценку значения $\Gamma_{n} \geq 10^{5}-10^{4} \mathrm{~s}^{-1}$. При меньших значениях $\Gamma_{n}$, в частности, для переходов с основного состояния необходимо использовать пространственно неоднородные решения уравнений (1), (2). Однако, как показано в [10], решения пространственно неоднородной задачи для основного состояния атома качественно согласуются с решениями пространственно однородной (для возбужденных состояний), в которых константа релаксации нижнего уровня $\Gamma_{n}$ заменяется средней пролетной шириной, определяемой поперечным размером $d$ светового пучка и наиболее вероятной скоростью частиц $v_{T}$. Поэтому далее рассматривается пространственно однородная задача.

Решение задачи зависит от направлений распространения световых волн и вектора напряженности магнитного поля $\mathbf{H}$, определяющего направление оси квантования. При распространении волн ортогонально направлению магнитного поля удается провести аналитическое рассмотрение задачи, а при распространении волн вдоль направления магнитного поля большое число независимых параметров и сложность формул делают аналитические решения практически бесполезными. В этом случае более информативными оказываются численные решения.

\section{1. Сильная и пробная волны в поперечном магнитном поле}

В этом случае рассматриваем задачу в системе координат с осью квантования (ось $Z$ ) вдоль направления напряженности сильного поля, полагая, что магнитное поле направлено вдоль оси $Z$. В этой системе координат для сильного поля разрешены переходы с изменением магнитного квантового числа $\Delta M=0$, а для пробного поля - с $\Delta M=0$ (при параллельных поляризациях оптических полей) и $\Delta M= \pm 1$ (при ортогональных поляризациях полей). Случай параллельных поляризаций полей сводится к двухуровневой задаче, которая исследована в [1]. Далее рассмотрим случай ортогональных поляризаций полей. Схема атомных переходов при действии световых полей в этом случае показана на рис. $1, a$. Из рисунка видно, что в рассматриваемой системе уровней образуются две связанные между собой подсистемы с $\Lambda$ - и $V$-типом переходов. При слабом пробном поле населенность верхнего магнитного подуровня с $M=0$ практически не зависит от интенсивности сильного поля и равна равновесному значению. Данное обстоятельство позволяет в этом случае провести аналитическое рассмотрение задачи на основе решения для одной подсистемы из $\Lambda$ - и $V$-типов переходов. Далее рассмотрим подсистему, образованную подуровнями $m, l$ (верхние) и $n, k$ (нижние) (рис. 1, a). Тогда решения системы уравнений (1), (2) для матрицы плотности движущегося со скоростью v атома ищем, согласно $[5,6]$, в виде

$$
\begin{gathered}
\rho_{m n}=r_{m n} \exp \left(-i \Omega_{1} t\right), \quad \rho_{m k}=r_{m k} \exp \left(-i \Omega_{1 \mu} t\right), \\
\rho_{k n}=r_{k n} \exp \left[i\left(\varepsilon+\omega_{k n}\right) t\right]
\end{gathered}
$$

в $\Lambda$-схеме и

$$
\rho_{l n}=r_{l n} \exp \left(i \Omega_{2 \mu} t\right), \quad \rho_{l m}=r_{l m} \exp \left[i\left(\varepsilon+\omega_{m l}\right) t\right]
$$

в $V$-схеме перехода. Здесь отстройки частот записаны с учетом допплеровского сдвига:

$$
\begin{gathered}
\Omega_{1}=\omega-\omega_{m n}-\mathbf{k v}, \quad \Omega_{1 \mu}=\omega_{\mu}-\omega_{m n}-\mathbf{k}_{\mu} \mathbf{v} \\
\Omega_{2 \mu}=\omega_{\mu}-\omega_{l n}-\mathbf{k}_{\mu} \mathbf{v}, \quad \varepsilon=\omega_{\mu}-\omega
\end{gathered}
$$

$\omega_{k n}$ и $\omega_{l m}$ - частоты запрещенных переходов $k-n$ и $l-m$. В стационарном случае из уравнений $(1),(2)$ в пренебрежении вкладом спонтанного переноса магнитной когерентности верхних уровней (обоснованность данного приближения показана в [10-12]) получаем следующие системы алгебраических уравнений на заселенности уровней $\rho_{i}$ и на коэффициенты поляризаций $r_{i j}$ :

$$
\begin{gathered}
\Gamma_{m} \rho_{m}=Q_{m}-v_{m n}\left(\rho_{m}-\rho_{n}\right)-v_{m k}\left(\rho_{m}-\rho_{k}\right)-2 \operatorname{Re}\left(i G^{*} r_{m n}\right), \\
\Gamma_{n} \rho_{n}=Q_{n}+A_{m n}\left(\rho_{m}+A_{l n} \rho_{l}\right)+v_{m n}\left(\rho_{m}-\rho_{n}\right) \\
+v_{l n}\left(\rho_{l}-\rho_{n}\right)+2 \operatorname{Re}\left(i G^{*} r_{m n}\right)+2 \operatorname{Re}\left(i G_{\mu}^{*} r_{l n}\right), \\
\Gamma_{l} \rho_{l}=Q_{l}-2 v_{l n}\left(\rho_{l}-\rho_{n}\right)+2 \operatorname{Re}\left(i G_{\mu}^{*} r_{n l}\right), \\
\Gamma_{k} \rho_{k}=Q_{k}-2 A_{m k}\left(\rho_{m}+2 v_{m k}\left(\rho_{m}-\rho_{k}\right)+2 \operatorname{Re}\left(i G_{\mu}^{*} r_{m k}\right) .\right.
\end{gathered}
$$

$$
\begin{gathered}
\left(\Gamma_{m n}-i \Omega_{1}\right) r_{m n}=-i G\left(\rho_{m}-\rho_{n}\right)-i G_{\mu} r_{m l}+i G_{\mu} R_{k n}, \\
\left(\Gamma_{m k}-i \Omega_{1 \mu}\right) r_{m k}=-i G_{\mu}\left(\rho_{m}-\rho_{k}\right)+i G_{\mu} r_{n k}, \\
{\left[\Gamma_{k n}+i\left(\varepsilon+\omega_{k n}\right)\right] r_{k n}=i\left(G_{\mu}^{*} r_{m n}-G r_{k m}\right),} \\
\left(\Gamma_{l n}-i \Omega_{2 \mu}\right) r_{l n}=-i G_{\mu}\left(\rho_{l}-\rho_{n}\right)-i G r_{l m}, \\
{\left[\Gamma_{l m}-i\left(\varepsilon+\omega_{m l}\right)\right] r_{l m}=i\left(G_{\mu} r_{n m}-G^{*} r_{l n}\right) .}
\end{gathered}
$$



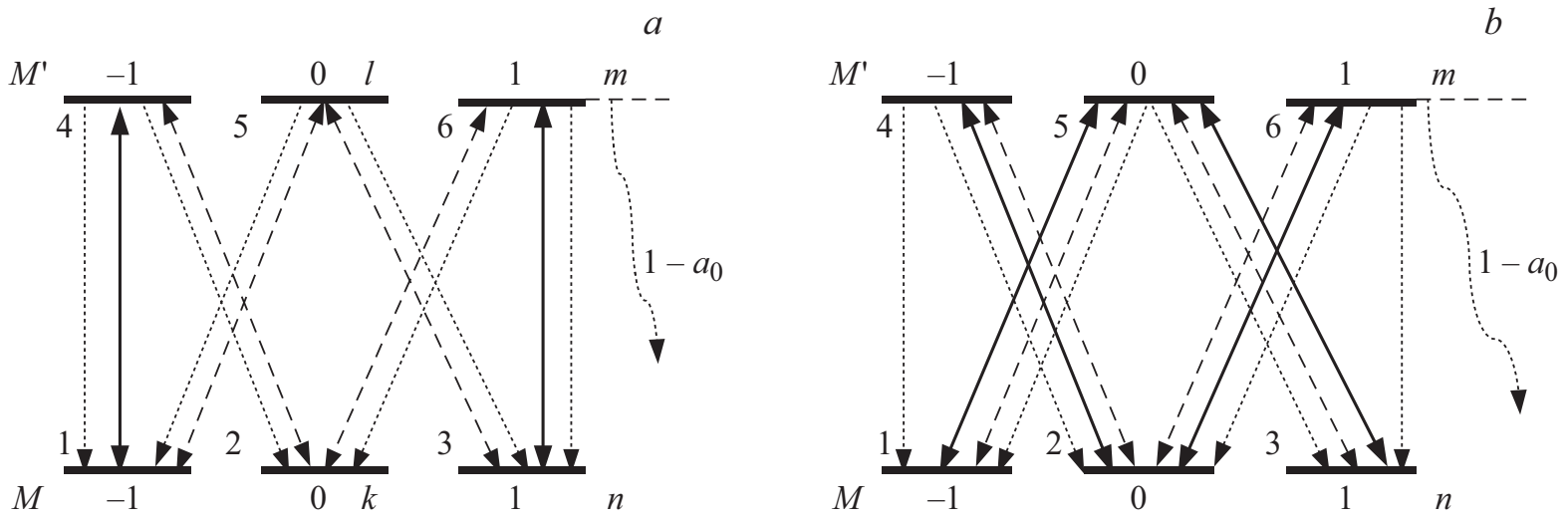

Рис. 1. Схема взаимодействия оптических полей с подуровнями перехода $J=1-J=1$ : сплошные линии - сильное поле, штриховые линии - пробное поле, пунктирные линии - спонтанный распад магнитных подуровней состояния $m$ на уровни состояния $n$ и прочие нижележащие уровни, $1-a_{0}$ - доля этого процесса; $(a)$ волны ортогональны направлению магнитного поля, $(b)$ волны параллельны направлению магнитного поля.

Коэффициент 2 в двух последних уравнениях системы (3) связан с равенством скоростей распада верхнего состояния по соответствующим каналам. Исключив из уравнений (4) амплитуды $r_{k n}$ и $r_{l m}$, получим следующие уравнения на амплитуды поляризаций на переходах $m-k$ и $l-n$ :

$$
\begin{gathered}
\left(\Gamma_{m n}-i \Omega_{1}+\frac{\left|G_{\mu}\right|^{2}}{\Gamma_{k n}+i\left(\omega_{k n}+\varepsilon\right)}+\frac{\left|G_{\mu}\right|^{2}}{\Gamma_{l m}+i\left(\omega_{m l}+\varepsilon\right)}\right) r_{m n} \\
=-i G\left(\rho_{m}-\rho_{n}\right)+\frac{G G_{\mu} r_{l n}^{*}}{\Gamma_{l m}+i\left(\omega_{m l}+\varepsilon\right)}+\frac{G G_{\mu} r_{k m}^{*}}{\Gamma_{k n}+i\left(\omega_{k n}+\varepsilon\right)}, \\
\left(\Gamma_{m k}-i \Omega_{1 \mu}+\frac{|G|^{2}}{\Gamma_{k n}-i\left(\omega_{k n}+\varepsilon\right)}\right) r_{m k} \\
=-i G_{\mu}\left(\rho_{m}-\rho_{n}\right)+\frac{G G_{\mu} r_{m n}^{*}}{\Gamma_{k n}-i\left(\omega_{k n}+\varepsilon\right)} \\
\left(\Gamma_{l n}-i \Omega_{2 \mu}+\frac{|G|^{2}}{\Gamma_{l m}-i\left(\omega_{m l}+\varepsilon\right)}\right) r_{l n} \\
=-i G_{\mu}\left(\rho_{l}-\rho_{n}\right)+\frac{G G_{\mu} r_{m n}^{*}}{\Gamma_{l m}-i\left(\omega_{m l}+\varepsilon\right)} .
\end{gathered}
$$

Решения систем уравнений (3)-(5) при произвольных полях сложны для анализа, поэтому далее рассмотрим случай слабого пробного поля $\left(\left|G_{\mu}\right|^{2} / \Gamma_{m n} \Gamma_{k n},\left|G_{\mu}\right|^{2} / \Gamma_{m n} \Gamma_{l m} \ll 1\right)$. Тогда уравнения (5) представимы в более простом виде:

$$
\begin{gathered}
\left(\Gamma_{m n}-i \Omega_{1}\right) r_{m n}=-i G\left(\rho_{m}^{0}-\rho_{n}^{0}\right), \\
\left(\Gamma_{m k}-i \Omega_{1 \mu}+\frac{|G|^{2}}{\Gamma_{k n}-i\left(\omega_{k n}+\varepsilon\right)}\right) r_{m k} \\
=-i G_{\mu}\left[\left(\rho_{m}^{0}-\rho_{k}^{0}\right)-\frac{|G|^{2}\left(\rho_{m}^{0}-\rho_{n}^{0}\right)}{\left[\Gamma_{k n}-i\left(\omega_{k n}+\varepsilon\right)\right]\left(\Gamma_{m n}+i \Omega_{1}\right)}\right],
\end{gathered}
$$

$$
\begin{aligned}
& \left(\Gamma_{l n}-i \Omega_{2 \mu}+\frac{|G|^{2}}{\Gamma_{l m}-i\left(\omega_{m l}+\varepsilon\right)}\right) r_{l n} \\
& =-i G_{\mu}\left[\left(\rho_{l}^{0}-\rho_{n}^{0}\right)-\frac{|G|^{2}\left(\rho_{m}^{0}-\rho_{n}^{0}\right)}{\left[\Gamma_{l m}-i\left(\omega_{m l}+\varepsilon\right)\right]\left(\Gamma_{m n}+i \Omega_{1}\right)}\right] .
\end{aligned}
$$

Здесь $\rho_{i}^{0}-$ решения системы уравнений, следующей из (3), при действии только сильного поля:

$$
\begin{gathered}
\Gamma_{m} \rho_{m}^{0}+v_{m n}\left(\rho_{m}^{0}-\rho_{n}^{0}\right)+v_{m k}\left(\rho_{m}^{0}-\rho_{k}^{0}\right) \\
+W_{m n}\left(\rho_{m}^{0}-\rho_{n}^{0}\right)=Q_{m}, \\
\Gamma_{n} \rho_{n}^{0}-A_{m n} \rho_{m}^{0}-A_{l n} \rho_{l}^{0}-v_{m n}\left(\rho_{m}^{0}-\rho_{n}^{0}\right)-v_{l n}\left(\rho_{l}^{0}-\rho_{n}^{0}\right) \\
-W_{m n}\left(\rho_{m}^{0}-\rho_{n}^{0}\right)=Q_{n}, \\
\Gamma_{l} \rho_{l}^{0}-2 v_{l n}\left(\rho_{l}^{0}-\rho_{n}^{0}\right)=Q_{l}, \\
\Gamma_{k} \rho_{k}^{0}-2 A_{m k} \rho_{m}^{0}-2 v_{m k}\left(\rho_{m}^{0}-\rho_{k}^{0}\right)=Q_{k},
\end{gathered}
$$

где

$$
W_{m n}=\frac{2|G|^{2} \Gamma_{m n}}{\Gamma_{m n}^{2}+\Omega_{1}^{2}} .
$$

Полагаем $\rho_{i}^{0}=N_{i}+r_{i}$, где $N_{i}$ - населенность $i$-го уровня в отсутствие сильного поля. Значения $N_{i}$ определяются из следующей алгебраической системы уравнений:

$$
\begin{gathered}
\Gamma_{m} N_{m}+v_{m n}^{0}\left(N_{m}-N_{n}\right)+v_{m k}^{0}\left(N_{m}-N_{k}\right)=Q_{m}, \\
\Gamma_{n} N_{n}-A_{m n} N_{m}-v_{m n}^{0}\left(N_{m}-N_{n}\right) \\
-A_{l n} N_{l}-v_{l n}^{0}\left(N_{l}-N_{n}\right)=Q_{n}, \\
\Gamma_{l} N_{l}+2 v_{l n}^{0}\left(N_{l}-N_{n}\right)=Q_{l}, \\
\Gamma_{k} N_{k}-2 A_{m k} N_{m}-2 v_{m k}^{0}\left(N_{m}-N_{k}\right)=Q_{k} .
\end{gathered}
$$

Здесь $v_{m n}^{0}=g_{n m}\left\langle N_{m}\right\rangle, v_{m k}^{0}=g_{k m}\left\langle N_{m}\right\rangle, v_{l n}^{0}=g_{l n}\left\langle N_{l}\right\rangle$. Коэффициенты $v_{i j}^{0}$ описывают эффект самонасыщения в среде в отсутствие сильного поля. 
Уравнения на коэффициенты $r_{i}$ следующие:

$$
\begin{gathered}
\Gamma_{m} r_{m}=\left(W_{m n}+v_{m n}\right)\left(r_{n}-r_{m}\right)+v_{m k}\left(r_{k}-r_{m}\right) \\
+\left(W_{m n}+\mu_{m n}\right) N_{n m}+\mu_{m k} N_{m k}, \\
\Gamma_{n} r_{n}=-\left(W_{m n}+v_{m n}\right)\left(r_{n}-r_{m}\right)-\left(W_{m n}+\mu_{m n}\right) N_{n m} \\
+A_{m n} N_{m}+A_{l n} N_{l}-v_{l n}\left(r_{n}-r_{l}\right)-\mu_{l n} N_{n l}, \\
\Gamma_{l} r_{l}=2 v_{l n}\left(r_{n}-r_{l}\right)+2 \mu_{l n} N_{n l}, \\
\Gamma_{k} r_{k}=v_{m k}\left(r_{m}-R_{k}\right)+A_{m k} N_{m}-\mu_{m k} N_{k m} .
\end{gathered}
$$

Здесь $N_{n m}=N_{n}-N_{m}, \quad N_{n l}=N_{n}-N_{l}, \quad N_{k m}=N_{k}-N_{m}$, $v_{m n}=v_{m n}^{0}+\mu_{m n}, v_{m k}=v_{m k}^{0}+\mu_{m k}, v_{l n}=v_{l n}^{0}+\mu_{l n}$, коэффициенты $\mu_{i j}$ описывают эффект самонасыщения, обусловленный только сильным полем. Отметим, что в рассматриваемой системе уровней (рис. $1, a$ ) скорости переноса между магнитными подуровнями связаны соотношениями: $A_{00}=0, A_{-1-1}=A_{-10}=A_{11}=A_{10} \cdot g_{M M^{\prime}}=g$; $v_{00}=0, v_{-1-1}=v_{11}=v_{10}=v_{-10}=v ; \mu_{00}=0, \mu_{-1-1}=$ $=\mu_{11}=\mu_{10}=\mu_{-10}=\mu$.

В общем случае решение системы уравнений (9) трудно анализируемое, поскольку детерминант системы представляет полином 4-й степени. Однако решение можно упростить, понизив ранг системы с использованием того факта, что в слабом пробном поле изменение населенности верхнего магнитного подуровня с $M=0$ (уровня $l$ ) обусловлено переносом населенностей с нижних подуровней под действием СИ. Можно показать, что заселенность этого уровня слабо отличается от равновесного значения, т.е. $\rho_{l}^{0} \approx N_{l}, r_{i} \approx 0$. В этом случае система (9) сводится к системе трех уравнений:

$$
\begin{gathered}
\left(\Gamma_{m}+2 v+W_{m n}\right) r_{m}-v r_{k}-\left(v+W_{m n}\right) r_{n} \\
=\left(W_{m n}+\mu\right) N_{n m}+\mu N_{k m}, \\
-\left(A_{s}+2 v\right) r_{m}+\left(\Gamma_{n}+2 v\right) r_{k}=-2 \mu N_{k m}, \\
2\left(\Gamma_{m}-A_{s}\right) r_{m}+2 \Gamma_{n} r_{n}+\gamma_{n} r_{k}=0 .
\end{gathered}
$$

Здесь $A_{s}=A_{m n}+A_{m k}, v_{m n}=v_{m k}=v_{l n}=v, \mu_{m n}=\mu_{m k}=\mu$. Решения системы (10) после выделения селективной части представимы в виде

$$
r_{i}=B_{i}+C_{i} \frac{\kappa_{3} \Gamma_{m n}^{2}}{\Gamma_{s 3}^{2}+\Omega_{1}^{2}},
$$

где $i=m, n, k ; \Gamma_{s 3} \equiv \Gamma_{m n} \sqrt{1+\kappa_{3}}-$ насыщенная ширина перехода. Параметр насыщения $\kappa_{3}$ определяется как

$$
\kappa_{3}=\kappa T_{3} / T_{m n}
$$

где

$$
\begin{gathered}
\kappa=2|G|^{2} T_{m n} / \Gamma_{m n}, \quad T_{m n}=\gamma_{m n} / \Gamma_{m} \Gamma_{n}, \\
T_{3}=\left[T_{m n}+\frac{A_{s}}{2 \Gamma_{m} \Gamma_{n}}+\frac{v}{\Gamma_{m} \Gamma_{n}}\left(2 T_{m n} \Gamma_{m}+1\right)\right] / F, \\
\gamma_{m n}=\Gamma_{m}+\Gamma_{n}-A_{s},
\end{gathered}
$$

$$
\begin{gathered}
F=1+v T_{1}+v^{2} T_{12}, \quad T_{1}=\left(2 \gamma_{m n}+A_{s} / 2+\Gamma_{m}\right) / \Gamma_{m} \Gamma_{n}, \\
T_{12}=\left(2 \gamma_{m n}+\Gamma_{n}\right) \Gamma_{m} \Gamma_{n}^{2} .
\end{gathered}
$$

Коэффициенты $B_{i}$ и $C_{i}$ задают амплитуды однородной полосы насыщения и селективной структуры на $i$-м уровне. Значения коэффициентов $B_{i}$ и $C_{i}$ определяются из решения системы уравнений (10), выражения для этих коэффициентов имеют следующий вид:

$$
\begin{gathered}
B_{m}=\left(\Gamma_{n} \Gamma_{m} F\right)^{-1}\left(2 \Gamma_{n}+3 v\right) \mu N_{n m}, \\
C_{m}=\left(\Gamma_{n} \Gamma_{m} F T_{3}\right)^{-1} N_{n m}\left[\left(\Gamma_{n}+2 v\right)\left(1-2 \mu T_{3}\right)+\mu\left(1+v T_{2}\right)\right], \\
B_{k}=-\left(\Gamma_{m} \Gamma_{n}^{2} F\right)^{-1}\left(\Gamma_{m}-A_{s}\right)\left(\Gamma_{n}+v\right) 2 \mu N_{n m}, \\
C_{k}=\left(\Gamma_{m} \Gamma_{n}^{2} F T_{3}\right)^{-1} N_{n m} \\
\times\left\{\Gamma_{n}\left(A_{s}+2 v\right)-2 \mu\left[\gamma_{m n}-T_{3}\left(\Gamma_{m}-A_{s}\right)\left(\Gamma_{n}+v\right)\right]\right\}, \\
B_{n}=-\left(\Gamma_{m} \Gamma_{n}^{2} F\right)^{-1} \mu N_{n m}\left(\Gamma_{m} \Gamma_{n}+2 v \gamma_{m n}\right), \\
C_{n}=\left(\Gamma_{m} \Gamma_{n}^{2} F T_{3}\right)^{-1} N_{n m} \\
\left.\times\left\{\Gamma_{m}-A_{s} / 2\right)\left(1+v / \Gamma_{n}\right)-\mu\left[1-T_{3}\left(\Gamma_{m}+v \gamma_{m n} / \Gamma_{n}\right)\right]\right\}, \\
B_{l}=0, \quad C_{l}=0 .
\end{gathered}
$$

Из выражений (12) следует, что СИ всегда увеличивает амплитуду однородной полосы на верхнем $m$ уровне и уменьшает ее на нижних уровнях. В случае закрытого перехода $\left(\Gamma_{m}=A_{s}\right)$ амплитуда полосы на нижнем уровне $k$ не изменяется. При этом оказывается, что всегда разности $B_{n, k}-B_{m}<0, C_{k}-C_{m}>0$, $C_{n}-C_{l}=C_{n}>0$, как это было во встречных волнах [4].

Рассмотрим спектр поглощения пробного поля, который задается его работой $P_{\mu}[5,6]$ как

$$
P_{\mu}=-2 h \omega_{\mu} \operatorname{Re}\left\langle i \sum_{i, k} G_{\mu_{i k}}^{*} r_{k i}\right\rangle .
$$

Отсюда, используя решения системы уравнений (6), получаем выражение для работы пробного поля:

$$
\begin{aligned}
& P_{\mu}=4 \hbar \omega_{\mu}\left|G_{\mu}\right|^{2} \operatorname{Re}\left\langle\frac{\Gamma_{k n}-i\left(\omega_{k n}+\varepsilon\right)}{\Delta_{1}}\right. \\
& \times\left[\left(\rho_{k}^{0}-\rho_{m}^{0}\right)-\frac{|G|^{2}\left(\rho_{n}^{0}-\rho_{m}^{0}\right)}{\left[\Gamma_{k n}-i\left(\omega_{k n}+\varepsilon\right)\right]\left(\Gamma_{m n}+i \Omega_{1}\right)}\right] \\
& +\frac{\Gamma_{l m}-i\left(\omega_{m l}+\varepsilon\right)}{\Delta_{2}} \\
& \left.\times\left[\left(\rho_{n}^{0}-\rho_{l}^{0}\right)-\frac{|G|^{2}\left(\rho_{n}^{0}-\rho_{m}^{0}\right)}{\left[\Gamma_{l m}-i\left(\omega_{l m}+\varepsilon\right)\right]\left(\Gamma_{m n}+i \Omega_{1}\right)}\right]\right\rangle,
\end{aligned}
$$

где

$$
\begin{aligned}
& \Delta_{1}=\left(\Gamma_{m k}-\Omega_{1 \mu}\right)\left[\Gamma_{k n}-i\left(\omega_{k n}+\varepsilon\right)\right]+|G|^{2}, \\
& \Delta_{2}=\left(\Gamma_{l n}-\Omega_{2 \mu}\right)\left[\Gamma_{l m}-i\left(\omega_{m l}+\varepsilon\right)\right]+|G|^{2},
\end{aligned}
$$


разности заселенностей определяются из соотношения (11) как

$$
\rho_{i}^{0}-\rho_{j}^{0}=N_{i j}+B_{i j}+C_{i j} \frac{\kappa_{3} \Gamma_{m n}^{2}}{\Gamma_{s 3}^{3}+\Omega_{1}^{2}},
$$

где $N_{i j}=N_{i}-N_{j}, B_{i j}=B_{i}-B_{j}, C_{i j}=C_{i}-C_{j}, i=k, n, l$, $j=m, n$, коэффициенты $B_{i}$ и $C_{i}$ вычисляются с помощью формул (12).

Далее основные закономерности проявления СИ рассмотрим в приближении первых нелинейных поправок по сильному полю (при $|G|^{2} / \Gamma_{m n} \Gamma_{n},|G|^{2} / \Gamma_{m n} \Gamma_{m} \ll 1$ ), т. е. насыщающее поле слабое. В этом приближении из соотношения (13) при усреднении по скоростям при большом допплеровском уширении $\Gamma_{m n} \sqrt{1+\kappa_{3}} \ll k v_{T}$ и отстройках частот полей $\Omega_{\mu} \ll k v_{T}, \Omega \ll k v_{T}$, можно получить выражение для работы пробного поля в виде

$$
P_{\mu}=4 \hbar \omega_{\mu}|G|^{2} \frac{\sqrt{\pi}}{k \nu_{T}}\left\{S+2|G|^{2} \operatorname{Re}\left[Y,\left(\delta_{\Lambda}, \delta V\right)\right]\right\},
$$

где $S=N_{k m}+N_{n l}+B_{k m}+B_{m l}$ - амплитуда подкладки контура линии поглощения,

$$
\begin{aligned}
& Y\left(\delta_{\Lambda}, \delta V\right)=\left(T_{3} C_{k m}+\frac{N_{n m}+B_{n m}}{\Gamma_{m n}+\Gamma_{m k}-\Gamma_{k n}}\right) \\
& \times \frac{1}{\Gamma_{m n}+\Gamma_{m k}-i \delta_{\Lambda}} \frac{N_{n m}+B_{n m}}{\Gamma_{m n}+\Gamma_{m k}-\Gamma_{k n}} \frac{1}{\Gamma_{k n}-i \delta_{\Lambda}} \\
& +\left(T_{3} C_{n l}+\frac{N_{n m}+B_{n m}}{\Gamma_{m n}+\Gamma_{l n}-\Gamma_{l m}}\right) \\
& \times \frac{1}{\Gamma_{m n}+\Gamma_{l n}-i \delta_{V}} \frac{N_{n m}+B_{n m}}{\Gamma_{m n}+\Gamma_{l n}-\Gamma_{l m}} \frac{1}{\Gamma_{l m}-i \delta_{V}}
\end{aligned}
$$

$\delta_{\Lambda}=\varepsilon+\omega_{k n}, \delta_{V}=\varepsilon+\omega_{m l}$. Величина $Y\left(\delta_{V}, \delta_{\Lambda}\right)$ определяет структуру спектра.

Важно отметить, что соотношения (14) и (15) получены при рассмотрении одной из подсистем перехода (см. выше). Для определения работы пробного поля на всем переходе необходимо учесть вклад его второй подсистемы, который определяется формально также формулами (14) и (15). Однако здесь частоты расщепления уровней магнитным полем $\omega_{k n}$ и $\omega_{m l}$ будут иметь противоположные знаки (рис. 1,a). Это приводит к расщеплению спектральных структур при расщеплениях уровней, превышающих их ширину.

При малых значениях расщеплений уровней магнитным полем $\omega_{k n}, \omega_{m l}<\Gamma_{n}$, разностях заселенностей $N_{n m}=N_{k m}=N_{n l}=\Delta N$ и константах релаксации уровней $\Gamma_{m n}=\Gamma_{l n}=\Gamma_{m k}=\Gamma, \Gamma_{k n}=\Gamma_{n}, \Gamma_{l m}=\Gamma_{m}$ имеем следующие соотношения:

$$
\delta_{\Lambda} \approx \delta_{V} \approx \varepsilon, \quad S=S_{0}=2 \Delta N+B_{k m}+B_{n l},
$$

$$
\begin{aligned}
& Y\left(\delta_{\Lambda} \delta_{V}\right)=Y(\varepsilon) \\
& =\left[T_{3}\left(C_{k m}+C_{n l}\right)+\left(\Delta N-B_{n m}\right)\left(\frac{1}{2 \Gamma-\Gamma_{n}}+\frac{1}{2 \Gamma-\Gamma_{m}}\right)\right] \\
& \times \frac{1}{2 \Gamma-i \varepsilon}-\frac{\Delta N-B_{n m}}{2 \Gamma-\Gamma_{n}} \frac{1}{\Gamma_{n}-i \varepsilon}-\frac{\Delta N-B_{n m}}{2 \Gamma-\Gamma_{m}} \frac{1}{\Gamma_{m}-i \varepsilon} .
\end{aligned}
$$

Из выражений (14), (16) следует, что спектр поглощения пробного поля состоит из допплеровской подкладки и трех резонансных структур с разными амплитудами и ширинами, центрированными на разности частот полей $\varepsilon=0$. Амплитуда подкладки определяется вкладами формируемых на переходах $m-k$ и $l-n$ полос однородного насыщения, зависящих от самонасыщения через разности коэффициентов $B_{k m}=B_{k}-B_{m}, B_{n l}=B_{n}-B_{l}$. Поскольку значения этих коэффициентов $\sim \mu T^{3}$, а разности коэффициентов $B_{k m}$ и $B_{n l}$ отрицательны $\left(B_{k m}<0\right.$, $\left.B_{n l}<0\right)$, то действие СИ с ростом его частоты всегда уменьшает амплитуду подкладки контура линии поглощения.

Первая резонансная структура (первое слагаемое в (16)) представляет лоренциан с полушириной 2Г и амплитудой, определяемой значениями релаксационных констант перехода и эффектом СИ (через коэффициенты $\left.C_{k m}, C_{n l}, B_{n m}\right)$. В отсутствие эффекта СИ значения разностей коэффициентов следующие: $B_{n m}=0, \quad T_{3} C_{n l}=N_{n m}\left(\Gamma_{m}-A_{s} / 2\right) / \Gamma_{m} \Gamma_{n}, \quad T_{3} C_{k m}=$ $=N_{n m}\left(A_{s}-\Gamma_{n}\right) / \Gamma_{m} \Gamma_{n}$. В этом случае амплитуда первой структуры положительна, и структура проявляется в виде пика поглощения. Максимум пика реализуется на закрытом переходе (при $\left.\Gamma_{m}=A_{m n}+A_{m k}\right)$. При действии СИ эта структура также проявляется в виде пика, но с меньшей амплитудой, поскольку для любых переходов $B_{n m}<0$, а разности коэффициентов $C_{k m}>0$ и $C_{n l}>0$, но их значения уменьшаются с ростом частоты самонасыщения. Отметим, что при соотношении констант $\Gamma_{m} \gg \Gamma_{n}$ основной вклад в формирования данной пиковой структуры, отсутствующей во встречных волнах [4], вносит $V$-схема перехода. Две другие структуры в (16) представляют собой лоренцианы с полуширинами верхнего $\Gamma_{m}$ - и нижнего $\Gamma_{n}$-уровней и образуют в работе пробного поля два провала с одинаковыми амплитудами. Действие СИ приводит к уменьшению амплитуд обоих провалов (через коэффициент $B_{n m}$ ), но не влияет на их ширины.

В результате сложения трех лоренцианов с разными ширинами и знаками амплитуд формируется сложный контур, который при соотношении констант релаксации $\Gamma_{m} \gg \Gamma_{n}$ содержит узкий (с полушириной $\Gamma_{n}$ ) провал в центре при $\varepsilon=0$. С усилением магнитного поля (при расщеплениях уровней $\Gamma_{m n} \geq \omega_{k n}, \omega_{m l} \geq \Gamma_{n}$ ) экстремумы структур, формирующих контур резонанса (15), сдвигаются по частоте согласно условиям резонанса, что приводит к расщеплению узкого провала на две компоненты и изменениям в крыльях контура. При этом 
действие СИ, как и при малых расщеплениях, проявляется в уменьшении амплитуд расщепленных компонент, но не влияет на ширины компонент. При расщеплениях уровней $\omega_{k n}, \omega_{m l}>\Gamma_{m n}$ вырождение уровней снимается, $\Lambda$ - и $V$-схемы перехода формируют отдельные компоненты спектра резонанса. Действие СИ в этих схемах рассмотрено нами в работе [1].

Таким образом, эффект самонасыщения в работе пробного поля на переходе между вырожденными уровнями с моментом $J=1$ при распространении волн ортогонально направлению магнитного поля в слабом насыщающем поле качественно подобен действию СИ в невырожденных $\Lambda$ - и $V$-типах переходов [1] и проявляется в изменении (уменьшении) амплитуды подкладки контура линии поглощения и амплитуд структур резонанса. В этом приближении СИ не влияет на ширины этих структур.

\section{2. Сильная и пробная волны в продольном магнитном поле}

В этом случае рассмотрение задачи проведем в системе координат с осью квантования вдоль направления распространения волн $(\mathbf{Z} \| \mathbf{H})$. В этой системе координат для обоих полей разрешены переходы с изменением магнитного квантового числа $\Delta M= \pm 1$ (рис. $1, b)$. Из рис. $1, b$ следует, что в рассматриваемой системе уровней образуются $\Lambda$ - и $V$-подсистемы, связанные между собой спонтанными переходами с изменением $\Delta M=0$.

В этом случае решения системы уравнений (1), (2) для матрицы плотности движущихся атомов ищем в следующем виде (приближение первой гармоники по $\varepsilon$ ): диагональные элементы как

$$
\begin{aligned}
\rho_{i} & =\rho_{i}^{0}+\rho_{i}^{+} \exp \left(i\left(\varepsilon t-\left(\mathbf{k}_{\mu}-\mathbf{k}\right) \mathbf{r}\right)\right) \\
& +\rho_{i}^{-} \exp \left(-i\left(\varepsilon t-\left(\mathbf{k}_{\mu}-\mathbf{k}\right) \mathbf{r}\right)\right)
\end{aligned}
$$

недиагональные элементы на разрешенных переходах как

$$
\begin{aligned}
\rho_{i k} & =R_{i k} \exp (-i(\omega t-\mathbf{k r}))+R_{i k}^{\mu} \exp \left(-i\left(\omega_{\mu} t-\mathbf{k}_{\mu} \mathbf{r}\right)\right) \\
& +R_{i k}^{s} \exp \left(-i\left(\omega_{\mu} t-\mathbf{k}_{s} \mathbf{r}\right)\right)
\end{aligned}
$$

а недиагональные элементы на запрещенном переходе как

$$
\begin{aligned}
\rho_{i k} & =r_{i k}^{0}+r_{i k}^{+} \exp \left(i\left(\varepsilon t-\left(\mathbf{k}_{\mu}-\mathbf{k}\right) \mathbf{r}\right)\right) \\
& +r_{i k}^{-} \exp \left(-i\left(\varepsilon t-\left(\mathbf{k}_{\mu}-\mathbf{k}\right) \mathbf{r}\right)\right),
\end{aligned}
$$

где $\varepsilon=\omega_{\mu}-\omega, \omega_{s}=2 \omega-\omega_{\mu}, \mathbf{k}_{s}=2 \mathbf{k}-\mathbf{k}_{\mu}$. Справедливость такого вида решений на переходе с $J=1$ в отсутствие самонасыщения показана нами в [10] при изменении интенсивностей пробной и насыщающей волн в широком диапазоне значений.

Тогда система уравнений для матрицы плотности (1), (2) в стационарном случае сводится к нелинейной алгебраической системе уравнений относительно коэффициентов $\rho_{i}^{0}, \rho_{i}^{ \pm}, R_{i k}, R_{i k}^{\mu}, R_{i k}^{s}, r_{i k}^{0}, R_{i k}^{ \pm}$. Учитывая эрмитовость этих коэффициентов, далее выписываем только независимые уравнения. При выводе уравнений рассматриваем случай однонаправленных волн с близкими по величине значениями волновых векторов $\mathbf{k}_{\mu} \approx \mathbf{k}$.

Из уравнения (1) следует система уравнений для заселенностей $i$-го подуровня нижнего $n$-состояния и $k$ го подуровня верхнего $m$-состояния:

$$
\begin{aligned}
& \Gamma_{n} \rho_{i}^{0}=Q_{i}+\sum_{k} A_{k i} \rho_{k}^{0}-\sum_{k} v_{i k}\left(\rho_{i}^{0}-\rho_{k}^{0}\right) \\
& +2 \operatorname{Re}\left(i \sum_{k} G_{i k} R_{i k}\right)+2 \operatorname{Re}\left(i \sum_{k} G_{i k}^{\mu} R_{i k}^{\mu}\right), \\
& (\Gamma+i \varepsilon) \rho_{i}^{+}=\sum_{k} A_{k i} \rho_{k}^{+} \\
& \quad+i \sum_{k}\left(G_{i k}^{\mu} R_{k i}-G_{k i} R_{i k}^{\mu}+G_{i k} R_{k i}^{s}\right), \\
& \Gamma_{m} \rho_{k}^{0}=Q_{k}-\sum_{i} v_{k i}\left(\rho_{k}^{0}--\rho_{i}^{0}\right) \\
& +2 \operatorname{Re}\left(i \sum_{i} G_{k i} R_{i k}\right)+2 \operatorname{Re}\left(i \sum_{i} G_{k i}^{\mu} R_{i k}^{\mu}\right), \\
& (\Gamma+i \varepsilon) \rho_{k}^{+}=i \sum_{i}\left(G_{k i} R_{i k}^{\mu}-G_{i k}^{\mu} R_{k i}^{\mu}-G_{i k} R_{k i}^{s}\right) .
\end{aligned}
$$

Из уравнения (2) получаем следующие системы уравнений на коэффициенты $R_{i k}, R_{i k}^{\mu}, R_{i k}^{s}$ для поляризаций на разрешенных переходах:

$$
\begin{gathered}
{\left[\Gamma_{m n}-i\left(\Omega_{i k}-\mathbf{k v}\right)\right] R_{i k}=-G_{i k}\left(\rho_{i}^{0}-\rho_{k}^{0}\right)} \\
-i G_{i k}^{\mu}\left(\rho_{k}^{+}-\rho_{i}^{+}\right)+i G_{i l} r_{l k}^{0}+i G_{i l}^{\mu} r_{i k}^{+}, \\
{\left[\Gamma_{m n}-i\left(\Omega_{i k}-\mathbf{k v}-\varepsilon\right)\right] R_{i k}^{s}=-i G_{i k}\left(\rho_{i}^{+}-\rho_{k}^{+}\right)+i G_{i l} r_{i k}^{+},} \\
{\left[\Gamma_{m n}-i\left(\Omega_{i k}^{\mu}-\mathbf{k v}\right)\right] R_{i k}^{\mu}=-G_{i k}\left(\rho_{i}^{+*}-\rho_{k}^{+*}\right)} \\
-i G_{i k}^{\mu}\left(\rho_{i}^{0}-\rho_{k}^{0}\right)+i G_{i l}\left(r_{l k}^{-}+i G_{i l}^{\mu} r_{l k}^{0} .\right.
\end{gathered}
$$

Здесь индексы $i$ и $k$ обозначают подуровни разных состояний, а индексы $l$ и $k$ обозначают подуровни одного состояния. В системах уравнений $(17)-(19)$ $\Gamma_{n}$ и $\Gamma_{m}-$ константы релаксации нижних и верхних уровней, $\Gamma_{m n}-$ однородная полуширина перехода, $\Omega_{i k}=\omega-\omega_{i k}$ и $\Omega_{i k}^{\mu}=\omega_{\mu}-\omega_{i k}-$ значения отстроек частот сильного и пробного полей от частот $\omega_{i k}$ переходов между магнитными подуровнями верхнего $m$ - и нижнего $n$-состояний, $\mathbf{k v}$ - допплеровский сдвиг частоты, $\mathbf{v}-$ вектор скорости атома.

Уравнения на коэффициенты $r_{i k}^{0}, r_{i k}^{ \pm}$для поляризаций на запрещенных переходах между магнитными подуровнями состояний (магнитной когерентности) будут следующими:

$$
\begin{aligned}
& \left(\Gamma_{i k}+i \omega_{i k}\right) r_{i k}^{0}=i\left(G_{i l} R_{i k}-C_{i k} R_{l k}^{*}\right. \\
& \left.+G_{i l}^{\mu} R_{l k}^{\mu}-G_{l k}^{\mu} R_{l i}^{\mu *}\right)-0.5 A_{m n} r_{l p}^{0}
\end{aligned}
$$




$$
\begin{aligned}
& {\left[\Gamma_{i k}+i\left(\omega_{i k}+\varepsilon\right)\right] r_{i k}^{+}} \\
& =i\left(G_{i l}^{\mu} R_{l k}-C_{l k} R_{l i}^{\mu *}+G_{i l} R_{l k}^{s}\right)-0.5 A_{m n} r_{l p}^{+} .
\end{aligned}
$$

Здесь $\omega_{i k}$ - частоты переходов между магнитными подуровнями нижнего $n$-, либо верхнего $m$-состояний, $\Gamma_{i k}$ - полуширины линий этих переходов (для нижнего состояния $\Gamma_{i k}=\Gamma_{n}$, для верхнего состояния $\Gamma_{i k}=\Gamma_{m}$ ), а индексы $l$ и $p$ относятся к верхнему состоянию. Для верхнего состояния в правых частях уравнений (20) будет отсутствовать последнее слагаемое, описывающее спонтанный перенос магнитной когерентности с верхнего состояния на нижнее. Скорость спонтанного переноса магнитной когерентности на рассматриваемом переходе равна $A_{m n} / 2[5]$.

Аналитические решения стационарной системы уравнений для матрицы плотности (17)-(20) сложны и трудно анализируемые даже в слабом пробном поле, поэтому эта система уравнений решалась численно при вариации констант релаксации уровней, параметра ветвления излучения $a_{0}$, относительной частоты самонасыщения (параметра $a_{m n}$ ) и интенсивностей оптических полей. В счете использовались, как и ранее в работах $[1,4,9]$, следующие релаксационные характеристики перехода: $\Gamma_{m}=5.5 \cdot 10^{7} \mathrm{~s}^{-1}, \Gamma_{n}=\left(10^{-2}-10^{-1}\right) \Gamma_{m}$, $\left.\Gamma_{m n}=\left(\Gamma_{m}+\Gamma_{n}\right) / 2\right), A_{m n}=a_{0} \Gamma_{m}$. Параметр ветвления $a_{0}$ варьировался в диапазоне $0.1-1$, а значения констант релаксации уровней изменялись от указанных выше до значений $\Gamma_{m}=\Gamma_{n}=\Gamma_{m n}$ при постоянной полуширине перехода $\Gamma_{m n}$. Ширины линий запрещенных переходов между магнитными подуровнями нижнего и верхнего состояний полагались равными значениям ширин уровней этих состояний. Допплеровская ширина линии принята равной $k v_{T}=5.2 \cdot 10^{9} \mathrm{~s}^{-1}$, диапазон изменения скоростей частиц при интегрировании составил $\pm 3 k v_{T}$ с шагом $\Delta v / v_{T}=10^{-3}-10^{-4}$. Значение параметра $a_{m n}$, характеризующего относительную частоту самонасыщения перехода, варьировалось согласно [2] в диапазоне 0-2.

Параметры насыщения сильного $\kappa_{s}$ и пробного $\kappa_{p}$ полей были выбраны в виде

$$
\begin{gathered}
\kappa_{s}=2(d E / 2 \sqrt{3} \hbar)^{2} \gamma_{m n} /\left(\Gamma_{m n} \Gamma_{m} \Gamma_{n}\right), \\
\kappa_{p}=2\left(d E_{\mu} / 2 \sqrt{3} \hbar\right)^{2} \gamma_{m n}\left(\Gamma_{m n} \Gamma_{m} \Gamma_{n}\right),
\end{gathered}
$$

где $E, E_{\mu}$ - напряженности циркулярных компонент сильного и пробного полей, $d-$ приведенный матричный элемент дипольного момента перехода $m-n$, $\gamma_{m n}=\Gamma_{m}+\Gamma_{n}-A_{m n}$. Значения пара метров насыщения изменялись в пределах $\kappa_{s}=0.1-50, \kappa_{p} \leq \kappa_{s}$.

Форма линии поглощения пробного поля (в расчете на один атом) определялась как

$$
\alpha / \alpha_{0}=-\Gamma_{m n}\left\langle\operatorname{Re}\left(i \sum_{i, k} R_{i k}^{\mu} G_{k i}^{\mu} /\left|G^{\mu}\right|^{2}\right)\right\rangle .
$$

Здесь обозначение $\langle\ldots\rangle$ означает усреднение по максвелловскому распределению скоростей частиц $\alpha_{0}=$ $=4 \pi \omega_{m n} d^{2} / c \hbar \Gamma_{m n}-$ сечение резонансного поглощения.

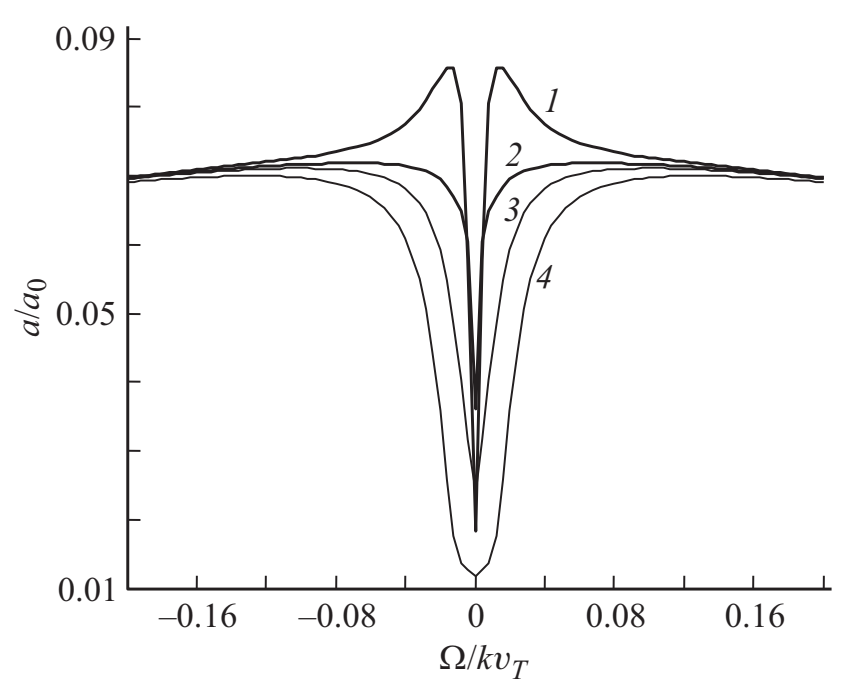

Рис. 2. Форма резонанса в отсутствие самонасыщения при $k_{s}=1, k_{p}=0.001, \Gamma_{n}=0.1 \Gamma_{m}$. Сплошные линии - ортогональные поляризации, пунктирные линии - параллельные поляризации полей при $a_{0}=1(1,4), 0.5(2,3)$.

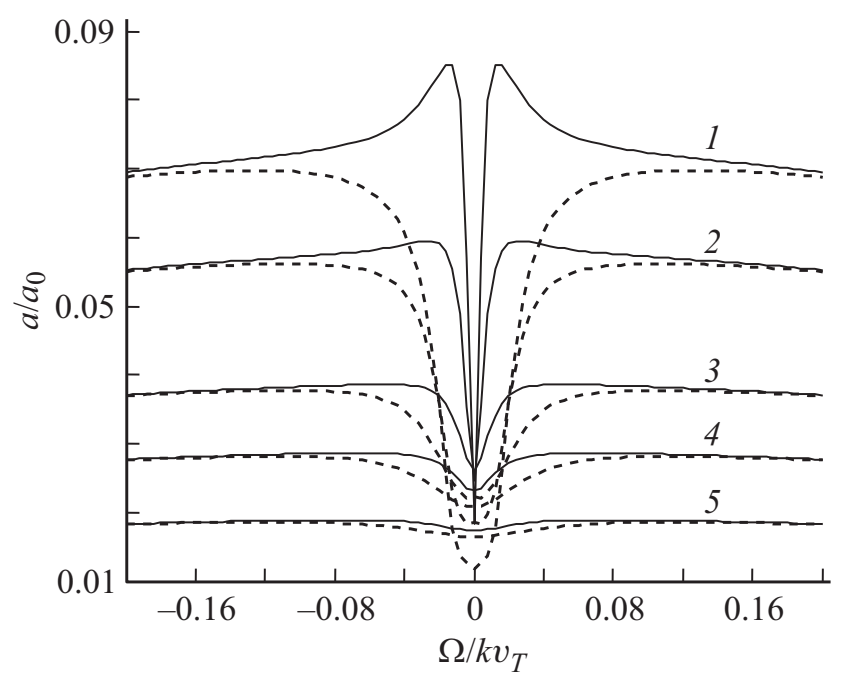

Рис. 3. Форма резонанса при самонасыщении: $a_{0}=1, k_{s}=1$, $k_{p}=0.001, \Gamma_{n}=0.1 \Gamma_{m}$. Сплошные линии - ортогональные поляризации, пунктирные линии - параллельные поляризации полей. $a_{m n}=0.01-0.1(1), 0.22(2), 0.35(3), 0.5$ (4), 0.75 (5).

\section{3. Форма резонанса в отсутствие эфффекта самонасыщения}

Результаты численного моделирования спектров насыщенного поглощения пробной волны в отсутствие СИ на исследуемом переходе показывают, что формы спектров зависят как от характеристик перехода (констант релаксации $\Gamma_{n}, \Gamma_{m}, \Gamma_{m n}$, параметра ветвления $\left.a_{0}\right)$, величины расщепления уровней и интенсивностей (параметров насыщения) сильной и пробной волн, так и от взаимной ориентации плоскостей поляризаций этих волн. При этом оказывается, что в сонаправленных волнах влияние параметра ветвления и направления взаимной 
ориентации поляризаций волн на спектры нелинейного поглощения более выражено, чем в случае встречно направленных волн [4].

На рис. 2 показаны характерные формы спектров насыщенного поглощения пробного поля при малом расщеплении уровней $\Omega_{H}<\Gamma_{n}$ в сонаправленных волнах, имеющих параллельные либо ортогональные линейные поляризации полей. Расчеты выполнены при соотношении констант релаксации уровней $\Gamma_{n}=0.1 \Gamma_{m}$ и значениях параметров насыщения полей $k_{s}=1, k_{p}=10^{-3}$ для двух типов переходов: открытого (с параметром ветвления $\left.a_{0}=0.5\right)$ и закрытого $\left(a_{0}=1\right)$.

При ортогональных поляризациях полей на допплеровском контуре линии поглощения пробной волны вблизи нулевых отстроек частот полей формируется нелинейный резонанс в виде узкого провала (пика просветления) на фоне широкого пика поглощения с небольшой амплитудой (кривые 1,2 ). В этом случае форма нелинейного резонанса подобна резонансу, наблюдаемому при распространении волн ортогонально оси квантования (разд. 2.1), а также в простой $\Lambda$-схеме переходов [5-8]. Расчеты показывают, что ширина узкой структуры резонанса, как и в разд. 2.1, определяется константой релаксации нижнего уровня $\Gamma_{n}$, а ширина пика - константой релаксации поляризации $\Gamma_{m n}$. При этом амплитуды пика и провала резонанса оказываются зависимыми от значений параметра ветвления $a_{0}$. $\mathrm{C}$ уменьшением параметра $a_{0}$ в диапазоне значений $a_{0}=1-0$ происходит как уменьшение глубины узкого провала, так и уменьшение амплитуды пиковой части резонанса вплоть до ее исчезновения (кривые 1,2). Значение параметра ветвления, при котором исчезает пиковая структура, находится в диапазоне $a_{0} \approx 0.75-0.6$ при изменении параметра насыщения сильного поля в пределах значений $\kappa_{s}=0.1-100$.

При параллельных поляризациях полей на контуре линии поглощения пробной волны вблизи нулевых отстроек частот полей также образуется нелинейный резонанс, но в виде широкого провала, внутри которого формируется узкий провал с небольшой амплитудой (кривые 3,4). Такая структура нелинейного резонанса наблюдается как на закрытом, так и на отрытом переходах. Как следует из аналитических и численных расчетов, основной провал резонанса обусловлен некогерентным процессом насыщения населенностей уровней перехода, его ширина определяется константой релаксации поляризации $\Gamma_{m n}$ и полевым уширением. Узкий провал обусловлен когерентным биением населенностей уровней в образующихся на переходе открытых двухуровневых схемах подобно [9]. В этом случае ширина узкой структуры определяется константой релаксации $\Gamma_{n}$ нижнего уровня, и с уменьшение величины $\Gamma_{n}$ происходит уменьшение ширины и рост амплитуды узкой структуры резонанса.

При параллельных поляризациях полей характеристики нелинейного резонанса также зависят от значений параметра ветвления $a_{0}$. Уменьшение параметра в диапазоне значений $a_{0}=1-0$ при постоянном параметре насыщения $\kappa_{s}$ приводит к уменьшению амплитуды и ширины основного провала и к увеличению контраста узкой структуры резонанса. При этом на закрытом переходе амплитуда и ширина провала значительно превосходят амплитуду и ширину провала на открытом переходе. Данная зависимость характеристик резонанса от значений параметра $a_{0}$ подобна наблюдаемой в системе двух уровней [9] и обусловлена тем фактом, что с увеличением открытости перехода (уменьшение значения $a_{0}$ ) заданная величина параметра насыщения $\kappa_{s}$ реализуется при меньших интенсивностях насыщающего поля. Уменьшение интенсивности насыщающего поля приводит к уменышению когерентной части резонанса и, согласно [9], к уменьшению его полной ширины и амплитуды при росте амплитуды и контраста узкой структуры.

Анализ результатов численных расчетов показал, что в амплитуду резонанса рис. 2 вносит вклад магнитная когерентность, индуцированная световыми полями непосредственно между подуровнями нижнего состояния, равный $\sim 15-20 \%$ при параллельных поляризациях и менее $10 \%$ при ортогональных поляризациях полей. При этом вклад переноса магнитной когерентности с уровней верхнего состояния, как и в [12], мал и составил $\leq 1 \%$.

При расщеплении уровней $\Omega_{H}>\Gamma_{n}$ происходит изменение спектров пробной волны рис. 2 (характер изменений зависит от направления поляризаций волн, подробнее см. [12]), а при расщеплениях $\Omega_{H}>\Gamma_{m n}$, когда снимается полностью вырождение уровней и отсутствуют вклады магнитной когерентности уровней, спектры нелинейного поглощения оказываются одинаковыми, не зависящими от направления поляризаций волн, и состоят из широкого провала с узким пиком пропускания вблизи разности частот $\varepsilon=0$ и двух узких структур с шириной нижнего уровня $\Gamma_{n}$, смещенных на удвоенную частоту расщепления уровней $\left(\varepsilon= \pm 2 \Omega_{H}\right)$.

Влияние интенсивности (параметра насыщения $\kappa_{s}$ ) сильной волны на характеристики нелинейного резонанса при любых поляризациях волн носит типичный характер [5-8]: с увеличением интенсивности происходит увеличение амплитуд и ширин как провалов, так и пиковой структуры резонанса. Влияние параметра насыщения $\kappa_{p}$ пробной волны проявляется иным образом: рост интенсивности пробной волны приводит к уменьшению амплитуд провала и пиковой части резонанса, а также к уменьшению допплеровского крыла линии подобно случаю встречных волн [4] как следствие насыщения пробным полем. При этом происходит уширение провала резонанса, причем уширение больше в случае параллельных поляризаций полей.

Изменение соотношения констант релаксации уровней (при $\Gamma_{n}>\Gamma_{m}$ ) при сохранении полуширины перехода $\Gamma_{m n}$ приводит к увеличению коэффициента поглощения в крыле линии ( 2 раза), уменьшению амплитуды 
пиковой части резонанса (при ортогональных поляризациях полей) и к увеличению амплитуды и ширины провала резонанса.

\section{4. Форма резонанса с учетом эффекта самонасыщения}

Учет эффекта самонасыщения в уравнениях (16)-(18) при численном моделирования формы резонанса насыщенного поглощения пробной волны показывает, что самонасыщение влияет как на амплитуды и контраст структур нелинейного резонанса (как и при аналитическом рассмотрении, разд. 2.1), так и на ширины этих структур. При этом эффект различается на закрытом и открытом переходах.

Характерное поведение формы нелинейного резонанса на закрытом переходе $\left(a_{0}=1\right)$ при малом расщеплении уровней $\Omega_{H}<\Gamma_{n}$ с учетом самонасыщения показано на рис. 3 при значениях параметров насыщения $k_{s}=1, k_{p}=10^{-3}$ для параллельных и ортогональных поляризаций полей. Из приведенных зависимостей следует, что действие СИ с ростом значения параметра $a_{m n}$ (относительной частоты самонасыщения) для обоих поляризаций полей приводит, во-первых, к уменьшению амплитуды крыльев допплеровского контура линии поглощения вследствие образования однородной полосы насыщения, во-вторых, к уменьшению амплитуд структур резонанса (как пика, так и провалов), а также их контрастов относительно допплеровской подкладки и, в-третьих, к уширению структур резонанса. Причем уширение полем СИ наиболее ярко проявляется при ортогональных поляризациях для узких структур резонанса, в то время как для широких структур эффект незначителен. В частности, в условиях рис. 3 уширение полем СИ при $a_{m n} \sim 0.5$ (кривая 4) превышает $100 \%$ ширины резонанса, связанного с нижним уровнем перехода. Подобное поведение формы нелинейных резонансов при действии самонасыщения наблюдается и на открытых переходах (с $\left.a_{0}<1\right)$. Значительное уширение узких структур резонанса полем СИ делает возможным экспериментальное измерение малых частот переноса, обусловленных эффектом самонасыщения.

Однако на закрытом переходе $\left(a_{0}=1\right)$ при любых поляризациях полей обнаруживаются особенности в проявлении эффекта СИ: если в крыле линии с ростом значения параметра $a_{m n}$ происходит последовательное уменьшение коэффициента поглощения, то вблизи центра линии при изменении параметра $a_{m n}$ сначала (в диапазоне значений $0.01-0.35$ ) наблюдается рост (кривые $1-3)$, а затем при $a_{m n}>0.35$ - последовательное уменьшение (кривые 4,5) коэффициента поглощения пробной волны.

В случае открытого перехода с $a_{0}=0.5$ аномального проявления самонасыщения в форме линии поглощения пробной волны не обнаруживается. Здесь эффект СИ с ростом значения параметра $a_{m n}$ приводит к последова- тельному уменьшению поглощения как в крыле, так и вблизи центра линии нелинейного резонанса.

Численные исследования показали, что обнаруженные различия в действии самонасыщения в спектрах насыщенного поглощения пробной волны вблизи центра линии на закрытом и открытом типах переходов обусловлены различием вкладов в работу пробного поля магнитной когерентности, индуцируемой полями линейно поляризованных световых волн на нижних уровнях этих переходов, при ее последующем разрушении некогерентным полем СИ.

Вклад магнитной когерентности уровней в форму нелинейного резонанса проявляется в узком диапазоне спектра вблизи центра линии как при параллельных, так и ортогональных поляризациях волн (рис. 2, кривые 1,4). При этом максимальный вклад реализуется на закрытом переходе при параллельных поляризациях волн $[11,12]$. Действие некогерентного поля СИ разрушает наведенную магнитную когерентность нижних уровней и приводит к росту коэффициента поглощения пробной волны вблизи центра линии на обоих типах переходов. Причем на закрытом переходе при малых частотах самонасыщения увеличение поглощения за счет разрушения магнитной когерентности уровней превалирует над уменьшением поглощения за счет образования однородной полосы самонасыщения (рис. 3, кривые $1-3$ ). При этом изменения в коэффициенте поглощения при параллельных поляризациях волн больше, чем при ортогональных поляризациях. С дальнейшим ростом частоты самонасыщения вклад полосы СИ начинает превалировать над вкладом магнитной когерентности, и поэтому поведение поглощения пробной волны вблизи центра линии оказывается таким же, как и в крыле линии (кривые 4,5).

На открытом переходе уже при малых частотах самонасыщения вклад однородной полосы насыщения в коэффициент поглощения превалирует над вкладом разрушения магнитной когерентности уровней полем СИ. Поэтому здесь характер проявления самонасыщения оказывается одинаковым как в центре, так и в крыльях линии поглощения.

В заключение раздела приведем оценки экспериментальных условий, при которых порождаются резонансы рис. 3. Отметим, что зависимости формы резонанса от значений параметра $a_{m n}$ по сути являются зависимостями от ширины пучка насыщающего поля при неизменных прочих параметрах и узком пучке пробного поля. При принятых в расчетах значениях параметров атомного перехода $\left(\lambda=6 \cdot 10^{-5} \mathrm{~cm}, A_{m n}=3 \cdot 10^{7} \mathrm{~s}^{-1}\right.$, $\left.\Gamma_{m}=5.5 \cdot 10^{7} \mathrm{~s}^{-1}, k v_{T}=5.2 \cdot 10^{9} \mathrm{~s}^{-1}\right)$ величина $a_{m n}$ связана с плотностью частиц на нижнем уровне и шириной пучка [2] как $a_{m n}=3 \cdot 10^{-12} N_{n} d_{\mathrm{ef}}\left(\mathrm{cm}^{-2}\right)$. При ширине пучка $d_{\mathrm{ef}}=1 \mathrm{~cm}$ и значениях параметра $a_{m n}=10^{-2}-0.75$ населенность нижнего уровня равна $N_{n}=3.3 \cdot 10^{9}-2.5 \cdot 10^{11}\left(\mathrm{~cm}^{-3}\right)$. При параметре насыщения сильного поля $\kappa_{s}=1$ оценка населенности верхнего уровня дает величину $N_{m} \sim N_{n} \Gamma_{m n} / 2 k v_{T} \sim$ 
$\sim 1.7 \cdot 10^{7}-1.3 \cdot 10^{9}\left(\mathrm{~cm}^{-3}\right)$. В этих условиях среда будет оптически тонкой поперек световых пучков, поскольку оптическая толщина согласно [2] определяется как $\alpha d_{\mathrm{ef}}=a_{m n} \Gamma_{m} /\left(\sqrt{\pi} A_{m n}\right) \approx 1.03 a_{m n}<1$.

Данные оценки определяют требования к экспериментальным условиям для проявления самонасыщения в спектре нелинейного резонанса при регистрации зависимости его формы от ширины насыщающего пучка.

\section{Заключение}

Таким образом, представленные результаты аналитических и численных исследований формы резонансов насыщенного поглощения в спектроскопии однонаправленных волн на переходах атомов с полным моментом уровней $J=1$ показывают их зависимость, как и во встречных волнах [4], от характеристик перехода (констант релаксации уровней $\Gamma_{m}, \Gamma_{n}$ и поляризации $\Gamma_{m n}$, параметра ветвления излучения $a_{0}$ ), интенсивностей сильной и пробной волн, а также от направления взаимной ориентации поляризаций этих волн. Причем в однонаправленных волнах в отличие от встречных волн направление ориентации поляризаций волн не только влияет на форму резонансов в количественном плане, но и способно качественным образом менять форму нелинейного резонанса. При параллельных поляризациях волн нелинейный резонанс проявляется на допплеровском контуре линии поглощения пробной волны вблизи нулевых отстроек частот в виде широкого провала и формируемого внутри провала узкого малоконтрастного пика просветления подобно случаю двухуровневой среды [9]. При ортогональных поляризациях волн нелинейный резонанс проявляется вблизи нулевых отстроек частот в виде узкого провала (пика просветления) на фоне широкого пика поглощения с небольшой амплитудой. Параметры провала и пика определяются значениями констант релаксации нижнего уровня $\Gamma_{n}$ и поляризации $\Gamma_{m n}$, значениями параметров насыщения полей, а также параметром ветвления $a_{0}$.

Важно отметить, что узкие структуры нелинейного резонанса на переходе между вырожденными состояниями атома в сонаправленных волнах формируются когерентными процессами, характер которых зависит от направления распространения волн по отношению к внешнему магнитному полю. При распространении ортогонально магнитному полю в случае параллельных поляризаций волн - это биение населенностей уровней в образующихся двухуровневых системах перехода $[8,9]$, а в случае ортогональных поляризаций волн - это НИЭФ или когерентное пленение населенностей в трехуровневых схемах перехода [5,7]. При распространении волн вдоль магнитного поля в формировании структур нелинейного резонанса наряду с указанными выше процессами оказывается важным еще и процесс индуцирования магнитной когерентности уровней ниж- него состояния перехода оптическими полями линейной поляризации.

Существенный вклад в форму нелинейных резонансов в однонаправленных волнах может вносить самонасыщение оптических переходов, явление в спектроскопии еще недостаточно изученное. Метод сонаправленных волн позволяет продвинуться в исследовании малых величин самонасыщения. Его действие, как показывают приведенные результаты, имеет как общие для всех переходов проявления, так и специфические. Общим является формирование в работе пробного поля полосы однородного насыщения, приводящей к уменьшению амплитуды контура линии поглощения, а также амплитуды и контраста населенностной части нелинейного резонанса при любых поляризациях волн. Специфика проявления самонасыщения переходов в сонаправленных волнах обнаруживается в особенностях уширения спектра когерентных структур нелинейного резонанса, а также в характере изменения амплитуд этих структур вследствие разрушения магнитной когерентности уровней перехода некогерентным полем СИ. Наиболее ярко это проявляется на закрытых типах переходов, где максимален эффект магнитной когерентности уровней (разд. 2.2.2).

\section{Благодарности}

Авторы признательны А.М. Шалагину за обсуждение работы и сделанные замечания.

\section{Финансирование работы}

Работа выполнена в рамках Программы СО РАН „Фундаментальные проблемы взаимодействия лазерного излучения с однородными и структурированными средами, перспективные технологии и устройства фотоники (II.10.2.) $)^{66}$.

\section{Конфликт интересов}

Авторы заявляют, что у них нет конфликта интересов.

\section{Список литературы}

[1] Сапрыкин Э.Г., Черненко А.А. // Опт. и спектр. 2017. T. 123. № 1. C. 94.

[2] Раутиан С.Г., Сапрыкин Э.Г., Черненко А.А. // Опт. и спектр. 2005. Т. 98. № 2. С. 292.

[3] Chernenko A.A., Rautian S.G., Saprykin E.G. // Laser Physics. 2005. V. 15. N 9. P. 1221.

[4] Раутиан С.Г., Сапрыкин Э.Г., Черненко А.А. // Опт. и спектр. 2005. Т. 98. № 3. С. 476; 2005. T. 99. № 6. С. 1014; 2008. T. 104. № 4. C. 630.

[5] Раутиан С.Г., Смирнов Г.И., Шалагин А.М. Нелинейные резонансы в спектрах атомов и молекул. Новосибирск: Наука, 1979.

[6] Rautian S.G., Shalagin A.M. Kinetic Problems of Nonlinear Spectroscopy. Amsterdam: North-Holland, 1991. 
[7] Летохов В.С., Чеботаев В.П. Нелинейная лазерная спектроскопия сверхвысокого разрешения. М.: Наука, 1990.

[8] Шалагин А.М. Основы нелинейной спектроскопии высокого разрешения. Новосибирск, 2008.

[9] Сапрыкин Э.Г., Черненко А.А., Шалагин А.М. // ЖЭТФ. 2016. T. 150. № 2. C. 238.

[10] Сапрыкин Э.Г., Черненко А.А., Шалагин А.М. // ЖЭТФ. 2014. T. 146. № 2. C. 229.

[11] Saprykin E.G., Chernenko A.A., Shalagin A.M. // Technical digest of MLPL-2016. Novosibirsk. 2016. P. 233.

[12] Сапрыкин Э.Г., Черненко А.А. // ЖЭТФ. 2018. Т. 154. № 2. C. 223. 